\title{
Factors affecting the regulation of pacing: current perspectives
}

\author{
This article was published in the following Dove Press journal: \\ Open Access Journal of Sports Medicine \\ 5 September 2014 \\ Number of times this article has been viewed
}

\author{
Alexis R Mauger \\ Endurance Research Group, School \\ of Sport and Exercise Sciences, \\ Faculty of Science, University of Kent, \\ Chatham, UK
}

\begin{abstract}
During prolonged dynamic and rhythmic exercise, muscular pain and discomfort arises as a result of an increased concentration of deleterious metabolites. Sensed by peripheral nociceptors and transmitted via afferent feedback to the brain, this provides important information regarding the physiological state of the muscle. These sensations ultimately contribute to what is termed "exercise-induced pain". Despite being well recognized by athletes and coaches, and suggested to be integral to exercise performance, this construct has largely escaped attention in experimental work. This perspective article highlights the current understanding of pacing in endurance performance, and the causes of exercise-induced pain. A new perspective is described, which proposes how exercise-induced pain may be a contributing factor in helping individuals to regulate their work rate during exercise and thus provides an important construct in pacing. Keywords: pain, exercise-induced pain, discomfort, exercise performance, self-paced
\end{abstract}

\section{Introduction}

Fatigue arising from repeated muscular contraction during exercise is a complex process, which is likely multifactorial and never absolute. ${ }^{1,2}$ Whilst the predominant mechanisms underpinning its etiology are well argued, it is generally accepted that in endurance exercise, maximizing speed or power output whilst limiting fatigue is the key determinant of success. ${ }^{3}$ Much of our basic understanding of how fatigue arises and affects performance has come from laboratory methodologies that require the subject to exercise to exhaustion at a fixed intensity or to produce a maximal voluntary contraction after a fatiguing task or intervention. ${ }^{4}$ In these examples, fatigue is more akin to an event (ie, task cessation) rather than a progressive process, or as an inability to produce maximal force (ie, maximal voluntary contraction), rather than the ability to maintain the repeated submaximal contractions that are consistent with endurance performance. Consequently, whilst these methods have been able to demonstrate metabolic factors (such as substrate depletion and accumulation of deleterious metabolites), ${ }^{5,6}$ and reduction in neural drive to the muscles and activation failure ${ }^{2}$ all likely contribute to the observed reduction in force or task cessation, they do not singularly explain endurance performance. Indeed, in true endurance performance, where time to completion is the measure of success, athletes rarely cease exercising and are not required to produce maximal contractions during or after the event. Rather, it is their ability to regulate their own work rate during the event, in order to stress their physiological capacity as close to this point as possible so that optimal performance is achieved without critically compromising their capacity during exercise. ${ }^{7}$ Consequently, endurance performance becomes more about the regulation of fatigue rather than a
Correspondence: Alexis R Mauger School of Sport and Exercise Sciences, University of Kent, Chatham,

Kent, ME4 4AG, UK

Tel +44 I6 34888997

Email lex.mauger@gmail.com 
terminal occurrence of it. This phenomenon has been termed pacing, and has previously been defined as "the manipulation of power output over an exercise bout, so as to balance energy expenditure and speed in a way that will allow completion of the activity to the best of the individual's capacity". ${ }^{8}$ The distinction between pacing and fixed intensity exercise has only started to receive significant attention in the last 15 years, during which time hundreds of important original research papers and reviews have been published on the subject. Because of its obvious role in endurance performance, this interest is likely to continue so that our understanding of this complex process will further improve. It is not the intention of this perspective piece to provide another review of the current pacing literature. Indeed, several excellent recent reviews have explored the most recent work in this area, and so we refer the reader to these for information concerning pacing and fatigue, ${ }^{9,10}$ decision-making, ${ }^{11,12}$ deception, ${ }^{13}$ central processes, ${ }^{14}$ and brain regions. ${ }^{15}$ Instead, the objective of this article is to present the case for the role of one largely ignored factor in the process of pacing, ie, exercise-induced pain and discomfort. It is the hope that this focus may encourage future work that can explore this proposal further.

\section{Current understanding of pacing}

There are a number of theories that explain the determinants of pacing in endurance performance, ${ }^{16-20}$ which have fairly consistent themes and largely originate from some of the basic propositions from (or objections to) the central governor model (CGM). Whilst research relating to the CGM defined much of what we now understand in pacing, the summary of pacing below draws from several different pacing theories. Consequently, the reader is directed to other articles for full explanations of the CGM, ${ }^{19}$ teleoanticipation, ${ }^{21}$ the rating of perceived exertion (RPE) template, ${ }^{22}$ and the hazard score. ${ }^{23}$ Taken together, these theories suggest that before exercise begins, an exercise template or schema of the estimated required work rate is calculated in a feedforward manner. This process requires knowledge of the exercise endpoint and is strengthened by prior experience of the same or similar activity. When exercise begins, the starting intensity is reliant on this preset schema; however, refinements to the individual's work rate are made once afferent feedback becomes available (ie, once a short period of "lag time" is complete). The exercise expectations (schema) are compared with the current physiological demand (afferent feedback) against the projected exercise duration (proximity of endpoint), which creates a sensation of the "difficulty" or "intensity" of exercise. This conscious sensation (or emotion, as defined by Noakes $)^{16}$ then drives the adjustments in work rate which are defined as pacing. A key foundation of the CGM is that a physiological reserve is maintained during all exercise, which serves a protective function by helping to prevent a catastrophic disturbance in homeostasis. The evidence to support such a physiological reserve is reasonably robust, as it is repeatedly shown that voluntary exercise performance can be significantly improved through a range of interventions which do not alter the exercise capacity of the muscle. ${ }^{24,25}$ However, the threshold of this physiological reserve appears to be fairly conservative, and so being able to access the reserve without critically disturbing homeostasis will result in an improved performance. Indeed, much of the supporting work for the CGM and pacing is based on demonstrating the existence of this physiological reserve.

As the exercise endpoint and prior experience are controllable, and are largely fixed variables between athletes, their importance with regard to endurance performance becomes fairly irrelevant. It may be that an athlete with a home advantage benefits from improved prior experience of a course, and so improves this feedforward aspect of pacing. However, in most circumstances, highly trained and elite athletes are likely to have similar prerace knowledge and experience. Therefore, the role of afferent feedback in regulating pacing is of primary interest for the athlete, coach, or scientist looking to improve performance, as this aspect is controllable during the race and different strategies can be employed to affect it. Afferent feedback is transferred through neural and humoral systems, ${ }^{26}$ and provides the brain with detailed information regarding the state of the exercising body. A large proportion of this feedback will arise from factors related to accumulation of metabolic byproducts, and so the role of markers of peripheral fatigue are likely directly involved in this feedback pacing process. ${ }^{16}$ As such, in endurance performance, the magnitude of this afferent feedback largely depends on the availability of oxygen at the muscle, and so the principal means of reducing afferent feedback for a given exercise intensity is for the athlete to have a high $\mathrm{VO}_{2 \max }$, a high lactate threshold, and good economy of movement. ${ }^{3}$ However, whilst these determinants of endurance performance are well known, they do not explain how the athlete is able to interpret afferent feedback in such a way that it is manifested as a conscious sensation (following its interaction with the pacing schema and proximity of the endpoint). Indeed, an athlete would be unlikely to be able to state the current concentration of intramuscular inorganic phosphate, their remaining glycogen storage, or their cardiac output. Thus, these constructs may be able to explain an athlete's "race pace", but they are not 
capable of explaining how the athlete is able to accurately match this work rate when external feedback (ie, power output or speed) is not available.

RPE has been suggested to be the sensory manifestation of these physiological components (and possibly their interaction with the feedforward process), and so is described as a key determinant of endurance performance. ${ }^{22}$ However, what actually produces this sensation is hotly contested and so defined differently between research groups. Indeed, depending on where an athlete is tested, they may be asked to rate their perceived exertion according to any combination of shortness of breath, effort to drive the legs, how hard the body is working, how heavy and strenuous the exercise is, muscle fatigue, and limb discomfort. Therefore, proposing RPE as a determinant of endurance performance is unhelpful because its physiological (or psychological) basis cannot be attributed to an objectively measurable construct, particularly when the construct/s cannot be agreed upon. This creates inconsistency between laboratories which limits the degree to which data regarding RPE can be compared between research groups. Furthermore, the lack of measurable mechanisms for RPE diminish its scientific "clout" as a recognized factor in endurance performance. Attempts have been made to partition aspects of the RPE scale into awareness of effort and physical sensations, ${ }^{27}$ although this Task Effort and Awareness scale presents an even more complex range of components than the RPE scale, and the conscious and subconscious aspects of the scale make it even harder to assess. It has been suggested that RPE can be explained solely by the sense of effort produced by the corollary discharge arising from motor output, ${ }^{28}$ which to some degree can be objectively measured. However, as other research groups do not describe RPE in this manner, it is hard to make firm conclusions and comparisons regarding these suggestions.

Whilst the different descriptions of RPE and lack of mechanistic data are limiting, there is generally wide agreement that RPE correlates well with changes in work rate and can be scaled according to exercise endpoint. ${ }^{22,29}$ Thus, regardless of the definition or mechanisms, its contribution to endurance performance is likely significant. However, it is not a sensation or concept that is recognized outside of exercise, and (compounded by the differences in scale description) is poorly understood by those who use it. Rather, if the conscious sensation that dictates pacing is so powerful, and present in those who have never seen an RPE scale, it is plausible that there is a well understood sensation accompanying intense exercise that is used to judge and moderate the work rate. By listening to athletes and coaches talk about their performance and training, it is apparent that exercise-induced pain and discomfort matches this description and has a major influence on regulation of pace. It is important to reaffirm at this point that pacing in endurance performance is likely to be a highly complex process, in which a multitude of factors contribute to the ultimate decision to upregulate or downregulate pace. Thus, the following discussion of exercise-induced pain and discomfort is not intended to supersede those factors in pacing that have already been acknowledged in the literature in the past. Rather, it is suggested that exercise-induced pain and discomfort contributes to this wider complex process and so provides a further mechanism in pacing that should be explored.

\section{Exercise-induced pain}

Pain has fascinated and puzzled clinicians and academics for centuries, and despite thousands of seminal original articles and review papers, there is still much to understand. For a comprehensive review of the area, the reader is referred to the excellent papers by Mense ${ }^{30}$ and Millan. ${ }^{31}$ Whilst the nociceptive mechanisms underpinning exercise-induced pain may be a relatively more finite area, the etiology of exercise-induced pain has not been agreed and it is suggested that it may arise from either (or a combination of) increased intramuscular pressure, release of noxious metabolites, or deformation of tissue associated with muscular contractions. A complete discussion of this is beyond the scope of the current article, so the reader is referred to a book chapter by Ellingson and $\mathrm{Cook}^{32}$ and a recent review paper by Dannecker and Koltyn ${ }^{33}$ for a thorough discussion on the subject.

Briefly, when exercise intensity is sufficient to create a metabolic disturbance in and around muscle, type III and type IV nociceptors are stimulated by mechanical and noxious pressure, heat, and deleterious metabolites that either sensitize or stimulate nociceptors. Type III nociceptors are stimulated by high threshold noxious pressure, which at a muscular level results in a dull aching or cramping pain. Stimulation of type IV fibers results in a similar sensation but there is a preferential response to noxious chemicals. ${ }^{34}$ Bradykinin, potassium, serotonin, and histamine act directly on type IV afferent nociceptors and sensitize type III afferent fibers. Hydrogen ions and prostaglandins do not directly activate nociceptors but have the important effect of sensitizing them. Increased exercise intensity results in an increased concentration of these noxious chemicals, so exercise-induced pain is tightly bound to exercise intensity. Indeed, the stimulus for pain threshold has been suggested to occur at $50 \%$ of $\mathrm{VO}_{2 \max }$ and to increase linearly with 
work rate. ${ }^{35}$ Therefore, exercise-induced pain becomes a familiar and well understood means by which to monitor the relative state of exercising muscle and cardiovascular strain. However, as suggested by the International Association for the Study of Pain, pain is always a subjective experience, emotions are always an element of pain, and the perception of pain is not always directly related to the magnitude of the nociceptive signal. Therefore, pain perceived by the athlete will always be relative and will likely be moderated by a variety of variables that are dependent on the nature of the exercise and the athlete's state of mind. This is an important point, because it may help to explain differences in exercise performance when other physiological variables are constant. There is evidence to suggest that athletes may be more tolerant to some forms of pain than nonathletes, and that regular and painful training may contribute to a decreased pain perception. ${ }^{36}$ Anshel and Russell ${ }^{37}$ have theorized that the ability of an athlete to tolerate exerciseinduced pain is a critical factor in endurance sports, and there appears to be agreement between athletes, coaches, and researchers that pain tolerance can limit certain types of athletic performance. ${ }^{37,38}$ In research, cycle ergometry has been consistently shown to cause naturally occurring muscle pain, ${ }^{35,39,40}$ although this measure is rarely collected during experimental research (but is sometimes integrated with RPE as feelings of "discomfort"). As pain is ultimately a physiological warning to remove the body from a potentially damaging situation or activity, the pain that arises as a result of intense exercise must convey a powerful drive to either stop the exercise or reduce its intensity so that pain decreases. As such, the ability to overcome this drive, or use it to refine pace, must be an important factor in endurance performance. This has largely been overlooked in research (with the exception of a few research groups, most notably $\mathrm{O}^{\prime}$ Connor et $\mathrm{al}^{38}$ ) and it is the intention of this perspective piece to highlight this omission.

\section{Perspectives}

This section will outline a current perspective for the role of exercise-induced pain in pacing and endurance performance. Whilst some of the following postulations have arisen as a result of published work, others are speculations based on currently unpublished data and anecdotal evidence, which are hoped to be investigated in future work.

It is proposed that the pain and discomfort arising from intense and repeated muscular contraction is used by an athlete to gauge their current exercise intensity (in combina- tion with a number of other factors, as alluded to previously) and indirectly, the physiological state of their exercising muscle. Hence, pain is an important construct in forming their pacing strategy. Changes in work rate are made so as to moderate the level of pain and discomfort perceived, with reductions in work rate to manage the increase in pain, and increases in work rate to increase pain to a level that the athlete is willing to endure. These suggestions are supported by work that has used analgesia during self-paced exercise to improve performance. ${ }^{24,41}$ In these studies, cyclists appear to exercise according to a set progression of pain, with changes in work rate used to maintain this. Through their ingestion of paracetamol, it was suggested that cyclists felt less pain for a given work rate, and so increased their power output to maintain the same level of pain. It should be noted that ingestion of other analgesics (aspirin and codeine) has not produced improvements in performance, ${ }^{42,43}$ which challenges the exercise-induced pain hypothesis. However, these studies utilized fixed intensity exercise, which is not comparable with self-paced exercise, ${ }^{44}$ and may be more to do with the differing mechanism of action of the analgesic. However, increasing pain by administration of naloxone has been shown to reduce time to exhaustion, ${ }^{45}$ which further demonstrates the complexity of this relationship and the difficulty of assessing it experimentally. The level of pain the athlete is prepared to engage in for a particular exercise will likely depend on a variety of factors, including motivation, competition, and prior experience. It is expected that increases in motivation and prior experience will reduce the subjective experience of exercise-induced pain or increase the willingness to endure it. Increased prior experience may explain why athletes are reported to have higher pain thresholds than nonathletes..$^{36}$ The limit of pain tolerance is potentially a key factor in determining the threshold for the physiological reserve, so that reducing the magnitude of the pain signal during exercise will result in a protective threshold that is pushed closer to the actual physiological capacity of the body, ie, the athlete will produce a higher work rate for the same level of pain, and so have a higher level of peripheral fatigue. The fact that a cyclist's performance is improved under conditions of mild analgesia in the presence of increased metabolic strain (increased heart rate and blood lactate) but no change in pain supports this concept. ${ }^{24} \mathrm{~A}$ critical variable in determining pain during exercise will likely be the exercise duration (proximity to endpoint), as intense pain may be tolerated for short periods of time, whereas moderate pain or discomfort will become unbearable if it continues for long periods. Consequently, moderate levels 
of induced pain may have little effect on short-duration or short-term voluntary contraction exercise, but elicit a large effect in long-duration exercise. Indeed, the effect of inducing experimental pain on short-duration exercise has produced equivocal results, ${ }^{46-49}$ whereas in exercise that lasts for long periods, increased or decreased pain frequently worsens or improves endurance performance, respectively. ${ }^{24,41,45}$ The difficulties associated with testing the exercise-induced pain hypothesis (ie, by experimentally increasing or decreasing pain) may contribute towards these differing findings. In line with previous research, trained individuals likely have a greater tolerance for exercise-induced pain, ${ }^{36}$ and may rely on this more to gauge their exercise intensity, and less so as a limiter of exercise performance. ${ }^{44}$ This may have links to associative and dissociative strategies that have previously been reported in running performance. ${ }^{50}$ This relationship may be difficult to assess in self-paced exercise, as trained individuals may report higher levels of pain simply because they are more willing to induce and endure this pain so that a higher work rate is maintained. Alternatively, it may be that they do not classify these sensations during exercise as pain because they are perceived differently. Thus, highly trained and elite athletes will likely be able to tolerate large amounts of exercise-induced pain for long time periods, or perceive exercise as less painful, whereas untrained individuals will have less tolerance to exercise-induced pain, and will use pain to more directly determine the threshold for the physiological reserve. Additionally, untrained individuals may have less willingness to engage in an exercise intensity that elicits pain and be more likely to cease exercise as a result of pain. Interventions that reduce the magnitude of pain perception during exercise will allow the athlete to maintain a higher work rate during the exercise and consequently improve their performance..$^{24,41}$ This effect may be stronger in lesser trained athletes as their physiological reserve will likely be more conservative than highly trained athletes. Interventions that remove all pain during exercise are likely to have no effect or a damaging effect on exercise performance, as this will disrupt the athlete's ability to monitor their exercise intensity and thus disrupt their pacing strategy. Indeed, by blocking all afferent feedback (including pain) during a $5 \mathrm{~km}$ time trial, Amann et $\mathrm{al}^{51}$ demonstrated that cyclists produced a positive pacing strategy of such magnitude that it induced severe peripheral fatigue in the later section of a time trial and caused severe ambulatory problems on completion. If such an experiment were to be completed with longer duration exercise, this blockade of afferent feedback (and pain) would likely elicit a greater negative effect on performance.

\section{Conclusion}

In summary, it is suggested that exercise-induced pain is one of several determinants of endurance performance, primarily because it facilitates awareness of the physiological state of muscle and consequently helps to regulate pace during moderate to long self-paced exercise. Its role and importance may differ depending on training status, the event, and other external factors. Whilst many other factors will be important in regulating pace, pain has previously not been widely addressed, so it is suggested that future work in endurance performance collects perceived pain as a standard perceptual measure so that its importance in exercise can be established.

\section{Disclosure}

The author reports no conflicts of interest in this work.

\section{References}

1. Fitts RH. Cellular mechanisms of muscle fatigue. Physiol Rev. 1994;74(1):49-94.

2. Gandevia SC. Spinal and supraspinal factors in human muscle fatigue. Physiol Rev. 2001;81(4):1725-1789.

3. Joyner MJ, Coyle EF. Endurance exercise performance: the physiology of champions. $J$ Physiol. 2008;586(1):35-44.

4. Ament W, Verkerke GJ. Exercise and fatigue. Sports Med. 2009;39(5): 389-422.

5. Coyle EF, Hagberg M, Hurley BF, Martin WH, Ehsani AA, Holloszy JO. Carbohydrate feeding during prolonged strenuous exercise can delay fatigue. J Appl Physiol. 1983;55(1):230-235.

6. Kent-Braun JA. Central and peripheral contributions to muscle fatigue in humans during sustained maximal effort. Eur J Appl Physiol Occup Physiol. 1999;80(1):57-63.

7. Mauger AR, Jones AM, Williams CA. Influence of feedback and prior experience on pacing during a 4-km cycle time trial. Med Sci Sports Exerc. 2009;41(2):451-458.

8. Mauger AR, Jones AM, Williams CA. The effect of non-contingent and accurate performance feedback on pacing and time trial performance in 4-km track cycling. Br J Sports Med. 2011;45(3): $225-229$.

9. St Clair Gibson A, De Koning JJ, Thompson KG, et al. Crawling to the finish line: why do endurance runners collapse? Implications for understanding of mechanisms underlying pacing and fatigue. Sports Med. 2013;43(6):413-424.

10. Knicker AJ, Renshaw I, Oldham AR, Cairns SP. Interactive processes link the multiple symptoms of fatigue in sport competition. Sports Med. 2011;41(4):307-328.

11. Smits BL, Pepping GJ, Hettinga FJ. Pacing and decision making in sport and exercise: the roles of perception and action in the regulation of exercise intensity. Sports Med. 2014;44(6):763-775.

12. Renfree A, Martin L, Micklewright D, St Clair Gibson A. Application of decision-making theory to the regulation of muscular work rate during self-paced competitive endurance activity. Sports Med. 2014;44(2): $147-158$.

13. Jones HS, Williams EL, Bridge CA, et al. Physiological and psychological effects of deception on pacing strategy and performance: a review. Sports Med. 2013;43(12):1243-1257.

14. Roelands B, de Koning J, Foster C, Hettinga F, Meeusen R. Neurophysiological determinants of theoretical concepts and mechanisms involved in pacing. Sports Med. 2013;43(5):301-311. 
15. Rauch HG, Schönbächler G, Noakes TD. Neural correlates of motor vigour and motor urgency during exercise. Sports Med. 2013;43(4): 227-241.

16. Noakes TD. Fatigue is a brain-derived emotion that regulates the exercise behaviour to ensure the protection of whole body homeostasis. Front Physiol. 2012;3:82.

17. Millet GY. Can neuromuscular fatigue explain running strategies and performance in ultra-marathons? The flush model. Sports Med. 2011;41(6):489-506.

18. Marcora S. Counterpoint: afferent feedback from fatigued locomotor muscles is not an important determinant of endurance exercise performance. J Appl Physiol. 2010;108(2):454-456.

19. Amann M, Secher NH. Point: afferent feedback from fatigued locomotor muscles is an important determinant of endurance exercise performance. J Appl Physiol. 2010;108(2):452-454.

20. Perrey S. Comments on point:counterpoint: afferent feedback from fatigued locomotor muscles is/is not an important determinant of endurance exercise performance. Predominance of central motor command in the regulation of exercise. J Appl Physiol. 2010;108(2): 458-468.

21. Ulmer HV. Concept of an extracellular regulation of muscular metabolic rate during heavy exercise in humans by psychophysiological feedback. Experientia. 1996;15(52):416-420.

22. Tucker R. The anticipatory regulation of performance: the physiological basis for pacing strategies and the development of a perceptionbased model for exercise performance. Br J Sports Med. 2009;43(6): 392-400.

23. de Koning JJ, Foster C, Bakkum A, et al. Regulation of pacing strategy during athletic competition. PLoS One. 2011;6(1):e15863.

24. Mauger AR, Jones AM, Williams CA. Influence of acetaminophen on performance during time trial cycling. J Appl Physiol. 2010;108(1): 98-104.

25. Swart J, Lamberts RP, Lambert MI, et al. Exercising with reserve: evidence that the CNS regulates prolonged exercise performance. $\mathrm{Br} \mathrm{J}$ Sports Med. 2008;43(10):782-788.

26. Critchley HD. Neural mechanisms of autonomic, affective, and cognitive integration. J Comp Neurol. 2005;493(1):154-166.

27. Swart J, Lindsay TR, Lambert MI, Brown JC, Noakes TD. Perceptual cues in the regulation of exercise performance - physical sensations of exercise and awareness of effort interact as separate cues. Br J Sports Med. 2012;46(1):42-48.

28. de Morree HM, Klein C, Marcora SM. Perception of effort reflects central motor command during movement execution. Psychophysiology. 2012;49(9):1242-1253

29. Faulkner J, Parfitt G, Eston R. The rating of perceived exertion during competitive running scales with time. Psychophysiology. 2008;45(6):977-985.

30. Mense S. Nociception from skeletal muscle in relation to clinical muscle pain. Pain. 1993;54(3):241-289.

31. Millan MJ. Descending control of pain. Prog Neurobiol. 2002;66(6): 355-474.

32. Ellingson LD, Cook DB. Physical activity and pain: neurobiological mechanisms. In: Ekkekakis P, editor. Routledge Handbook of Physical Activity and Mental Health. New York, NY, USA: Routledge; 2014
33. Dannecker EA, Koltyn KF. Pain during and within hours after exercise in healthy adults. Sports Med. 2014;44(7):921-942.

34. Marchettini P, Simone DA, Caputi G, Ochoa JL. Pain from excitation of identified muscle nociceptors in humans. Brain Res. 1996;18(740): 109-116.

35. Cook DB, O’Connor PJ, Eubanks SA, Smith JC, Lee M. Naturally occurring muscle pain during exercise: assessment and experimental evidence. Med Sci Sports Exerc. 1997;29(8):999-1012.

36. Scott V, Gijsbers K. Pain perception in competitive swimmers. BMJ. 1981;11(283):91-93.

37. Anshel MH, Russell KG. Effect of aerobic and strength training on pain tolerance, pain appraisal and mood of unfit males as a function of pain location. J Sports Sci. 1994;12(6):535-547.

38. O’Connor PJ. Psychological aspects of endurance performance. In: Shephard RJ, Astrand PO, editors. Endurance in Sport. Oxford, UK: Blackwell Science; 1992.

39. Borg G, Ljunggren G, Ceci R. The increase of perceived exertion, aches and pain in the legs, heart rate and blood lactate during exercise on a bicycle ergometer. Eur J Appl Physiol Occup Physiol. 1985;54(4):343-349.

40. O'Connor PJ, Cook DB. Exercise and pain: the neurobiology, measurement, and laboratory study of pain in relation to exercise in humans. Exerc Sport Sci Rev. 1999;27:119-166.

41. Foster J, Taylor L, Chrismas BC, Watkins SL, Mauger AR. The influence of acetaminophen on repeated sprint cycling performance. Eur J Appl Physiol. 2014;114(1):41-48.

42. Hudson GM, Green JM, Bishop PA, Richardson MT. Effects of caffeine and aspirin on light resistance training performance, perceived exertion, and pain perception. J Strength Cond Res. 2008;22(6):1950-1957.

43. Ray CA, Carter JR. Central modulation of exercise-induced muscle pain in humans. J Physiol. 2007;15(585):287-294.

44. Mauger AR. Fatigue is a pain-the use of novel neurophysiological techniques to understand the fatigue-pain relationship. Front Physiol. 2013;13(4):104.

45. Sgherza AL, Axen K, Fain R, Hoffman RS, Dunbar CC, Haas F. Effect of naloxone on perceived exertion and exercise capacity during maximal cycle ergometry. J Appl Physiol. 2002;93(6):2023-2028.

46. Graven-Nielsen T, Lund H, Arendt-Nielsen L, Danneskiold-Samsøe B, Bliddal $\mathrm{H}$. Inhibition of maximal voluntary contraction force by experimental muscle pain: a centrally mediated mechanism. Muscle Nerve. 2002;26(5):709-712.

47. Khan SI, McNeil CJ, Gandevia SC, Taylor JL. Effect of experimental muscle pain on maximal voluntary activation of human biceps brachii muscle. J Appl Physiol. 2011;111(3):743-750.

48. Lisse JR, Macdonald K, Thurmond-Anderle ME, Fuchs JE. A doubleblind placebo controlled study of acetylsalicylic acid (ASA) in trained runners. J Sports Med Phys Fitness. 1991;31(4):561-564.

49. Roi GS, Garagiola U, Verza P, et al. Aspirin does not affect exercise performance. Int J Sports Med. 1994;15(5):224-227.

50. Morgan WP, Pollack ML. Psychological characterization of the elite distance runner. Ann N Y Acad Sci. 1977;301:382-403.

51. Amann M, Proctor LT, Sebranek JJ, Pegelow DF, Dempsey JA. Opioid-mediated muscle afferents inhibit central motor drive and limit peripheral muscle fatigue development in humans. J Physiol. 2009; 587 Pt 1:271-283.
Open Access Journal of Sports Medicine

\section{Publish your work in this journal}

Open Access Journal of Sports Medicine is an international, peer-reviewed, open access journal publishing original research, reports, reviews and commentaries on all areas of sports medicine. The manuscript management system is completely online and includes a very quick and fair peer-review system.

\section{Dovepress}

Visit http://www.dovepress.com/testimonials.php to read real quotes from published authors. 